\title{
Experimental Model Validation and Fatigue Behaviour of Cold-Formed High Strength Steel ${ }^{+}$
}

\author{
S. Gothivarekar 1,2, , S. Coppieters ${ }^{1,2}$, R. H. Talemi ${ }^{1,2}$ and D. Debruyne ${ }^{1,2}$ \\ 1 Department of Materials Engineering, KU Leuven, 3001 Leuven, Belgium; \\ sam.coppieters@kuleuven.be (S.C.); reza.hojjatitalemi@kuleuven.be (R.H.T.); \\ dimitri.debruyne@kuleuven.be (D.D.) \\ 2 Mechanics of Materials, Products and Processes (MeM2P), KU Leuven, 9000 Gent, Belgium \\ * Correspondence: sanjay.gothivarekar@kuleuven.be \\ † Presented at the 18th International Conference on Experimental Mechanics, Brussels, Belgium, 1-5 July 2018.
}

Published: 4 May 2018

\begin{abstract}
In the present paper, fatigue fracture behaviour of cold-formed High Strength Steel (HSS) S690QL are investigated. S690QL is often employed, in a pre-deformed state, for load-bearing applications, where cyclic service loads can be critical. Bending processes can induce residual plastic strain in bent areas and this changes the fatigue behaviour significantly. Traditional uniaxial dogbone testing cannot represent this multi-axial phenomenon properly and for this purpose a benchmark specimen is proposed, that is first bent and after subjected to fatigue loading. The bending process is modelled with a quasi-static Finite Element (FE) model, performed in the commercial code Abaqus. To validate the numerical model, a stereo Digital Image Correlation (DIC) set-up is used, capable of measuring the residual plastic strains in the specimen. The present article covers the validation of a cold-forming process followed by a preliminary fatigue analysis of S690QL.
\end{abstract}

Keywords: cold-forming process; Stereo DIC; numerical modelling; HSS; fatigue

\section{Introduction}

In recent years, effective and efficient manufacturing technologies have paved the way of sophisticated, modern, structural design. The importance of innovation in this field is reflected in numerous advances towards materials science \& engineering, with applications of increasing magnitude and complexity, [1]. For this reason, High Strength Steels (HSS) are often preferred for their desirable advantages, such as a high strength-to-weight ratio and increased load capacity. Typical applications include heavy-duty machinery and different types of cranes such as mobile, telescopic and tower cranes. These structures often require a square or rectangular cross sections, derived from various forming processes. However, secondary forming processes can induce a considerable amount of plastic deformation that weakens the material, resulting in stress concentrations and hot-spots. Formed, structural components are then subjected to fluctuating service loads, causing fatigue loading conditions that may initiate microscopic cracks, ultimately leading to catastrophic failure. Therefore, accurate fatigue characterisation of pre-deformed HSS has become a major concern for adequate structural design. Since residual stresses, induced by metalworking, have a significant influence on the cyclic behaviour, they must be accounted for.

Several authors have studied residual stresses and the effect on fatigue of construction steels as well as HSS. Talemi et al. [2] considered the effect of pre-deformation on the fatigue life of a thick, high strength S700MC steel plate. The study showed the feasibility of lock-in thermography to monitor the crack growth of low-cycle fatigue tests and finite elements analysis to provide information of the multi-axial stress gradient in the material. Vucko et al. [3] investigated the internal 
and effective stress evolution, on a microstructural level, of S690QL during fatigue loading. They have found that the internal stress depends on the formation of cells whereas athermal effective stress is mainly caused by the reduction of dislocation density. Shimatana et al. [4] studied the effect of residual stresses on the high cycle fatigue behaviour of HSS. The study has shown that crack propagation under low cycle fatigue loading conditions, was mainly caused by surface cracks, while high cycle fatigue loading conditions lead to subsurface crack growth. It is clear that up till now a variety of studies has been performed on forming processes and fatigue behaviour of HSS, however only a few investigate the occurrence of both phenomena simultaneously. In this research, the deformation of cold-formed S690QL is investigated, both numerically and experimentally. Therefore a validated FE-model is employed to simulate the bending process and predict the residual stress state of the bent area. Further an attempt is made to characterise the fatigue properties of the deformed material.

\section{Experimental Procedure}

\subsection{Materials and Specimen}

The investigated steel grade S690QL, is a structural (S) steel with a minimum guaranteed yield strength of $690 \mathrm{MPa}$, that has been quenched (Q) and tempered to achieve greater strength and durability. It is in compliance with EN 10025-6 where a minimum impact resistance at a low notch temperature is prescribed (L) [5]. An overview of the general mechanical properties of S690QL are tabulated in Table 1.

Table 1. Mechanical properties of S690QL in Rolling Direction [1].

\begin{tabular}{cccc}
\hline S690QL & $\boldsymbol{\sigma}_{\mathbf{y}}[\mathrm{MPa}]$ & $\boldsymbol{\sigma}_{\text {UTS }}[\mathrm{MPa}]$ & $\boldsymbol{\varepsilon}[\%]$ \\
\hline RD-AVG & $748.4 \pm 1.4$ & $804.4 \pm 1.5$ & $37.7 \pm 3.6$ \\
\hline
\end{tabular}

HSS components used for heavy-duty machinery and civil constructions are usually wide enough to ensure plain strain conditions. For this reason, the benchmark specimen was designed to be wider than a traditional dogbone specimen. The samples are cut by water jet from a 12-mm thick plate and then bent using an air-bending set-up displayed in Figure 1a. For DIC-measurements a tensile test machine (Zwick Roell Z250kN) is employed to deform the specimen to $\pm 90^{\circ}$ angles, resulting in a V-shape. Fatigue benchmark specimens consist of two bent specimens, that have been welded together at the top, to achieve a symmetrical configuration as shown in Figure 1c.
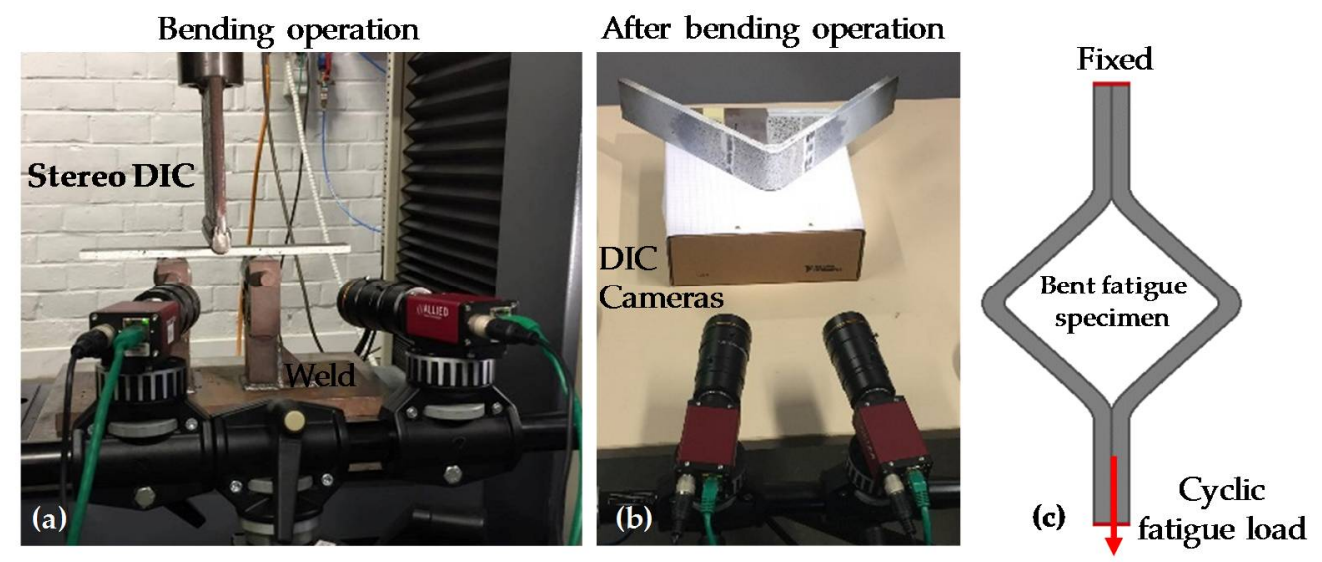

Figure 1. (a) Air-bending setup; (b) Incremental measurement of bottom side; (c) V-fatigue specimen.

\subsection{Measurement Technique}

DIC is a full field measurement technique which is used in this investigation to analyse the residual stress distribution of the specimen after the bending process and to validate the FE-model. 
DIC is an optical numerical method, capable of determining complex displacement and strain fields at the material surface [6]. Since in- and out-of-plane deformation occurs near the bending root, a stereo DIC-set-up is required. Allied Vision Manta G609 CCD sensors were used, with lenses of focal length of $25 \mathrm{~mm}$. Both front and bottom surfaces were measured using two separate 3D stereo setups. A first setup was placed near the lower testing area of the tensile machine, perpendicular to the front side of the specimen. The second set-up, in the vicinity of the experiment, measured the bottom side incrementally as shown in Figure 1b. For post-processing, a ZNSSD (Zero Normalized Sum of Squared Differences) criterion has been used, since it can control the effects of offset and scaling of light. An affine shape function is sufficient to deliver accurate results. A subset of 25 and step size of 8 pixels, resulted from a performance analysis module of MatchID. There, different post-processing settings are compared and obtained to derive accurate results. Once the correlation was complete, displacement and strain fields were visualized for all load steps and was used afterwards to validate the developed FE-model, as is elaborated in section 4.

\subsection{V-Fatigue Test}

A major concern for many HSS applications, subjected to cyclic loads, is the accurate prediction of the fatigue fracture behaviour. According to literature [7] a few standard testing methods exist to identify this behaviour. However, these standard tests do not incorporate the effect of a forming process or multiaxial loading. To this purpose, a method has been adopted from previous research [2] to characterise the fatigue resistance of a V-shaped benchmark specimen. The geometry consists of two bent samples that are welded together at the ends. A preliminary fatigue test has been performed for bent S690QL. A constant amplitude, fatigue loading of $50 \mathrm{kN}$ was applied with a loading ratio and frequency of $\mathrm{R}=0.1$ and $f=25 \mathrm{~Hz}$, respectively.

This causes compressive stress to build up on the outside of the bend and critical tensile stress on the inside of the bent area. From literature [3] it is found that tensional stresses are generally more critical for fatigue since they result in higher stress intensity and reduced growth resistance near the material surface. For this reason, micro cracks are most likely to initiate from the inside of the bending root. This part of the research was focussed on the feasibility of an experimental V-fatigue test to derive preliminary data for cold-formed S690QL.

\section{FE-Modelling}

FE analysis has been used in this study to predict the residual strain and bend angle of the specimen after bending. Since a DIC speckle pattern of spray paint in the bending root would deteriorate due to contact with the punch, a validated FE-model was employed. A 3D model was created in Abaqus v6.13 standard and consists of three parts, a deformable solid specimen and two discrete rigid shells, a cylindrical support and punch. Because of symmetry along XY and YZ plane, only a quarter of the specimen was bent by the two rigid parts. The meshed assembly can be seen in Figure 2a. An isotropic material was assumed with a Young's modulus E = $210 \mathrm{GPa}$ and a Poisson ratio $v=0.33$. The plastic anisotropy of S690QL was thoroughly investigated by Denys et al. [8]. This study showed that plastic anisotropy is weak, especially at larger plastic deformation. As consequence, S690QL material was modelled by a calibrated von Mises yield criterion.

The solid curve in Figure $2 b$ shows the true stress-true strain curve acquired via a quasi-static tensile test. It can be inferred that initial yielding starts at $760 \mathrm{MPa}$, while maximum uniform strain is limited to about $5 \%$. This region is imperative to accurately reproduce initial yielding and the final material state after spring back. The post-necking strain hardening behaviour, represented by the dashed line in Figure $2 b$, is required to simulate the large plastic deformation during bending. ThIs was modelled using a p-law investigated in previous research [9]. The problem was solved in one initial and two general non-linear static steps. During the first step, the punch had a vertical displacement downwards. In the next step, the punch returned upwards and loses contact, allowing the specimen to spring back to its final angle. To achieve the desired outline, the precise amount of elastic springback must be accounted for. Through iteration of the maximum displacement, a value of $42 \mathrm{~mm}$ was found corresponding to a bend angle of $90^{\circ}$, after the springback. Values of $3.3^{\circ}$ to $3.4^{\circ}$ 
have been predicted for the bent specimen. At the end of the second step, residual strains were analysed and are discussed in the following section.
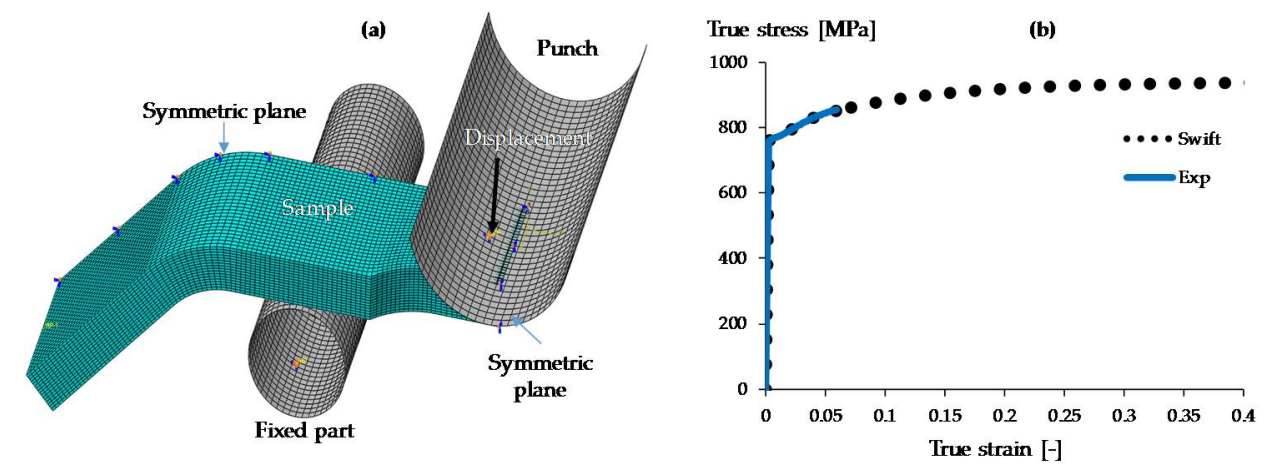

Figure 2. (a) Assembly of 3D-model with mesh and boundary conditions; (b) Material model.

\section{Model Validation}

To validate the FE-model, a DIC measurement was performed on the bent specimen, as explained in the experimental section. The commercial code MatchID 2018 was used to correlate the experimental images and produce full-field strain measurements. The results from the FEA are compared here with the experimental observations, by extracting data from the area of interest. Since the maximum principal strain measured, mostly consists of tensional strain in x-direction, $\varepsilon_{x x}$ of the DIC experiment was compared with the results of the FE-model. Figure 3 plots the total longitudinal strain $\varepsilon_{x x}$ along the bottom side of the specimen. Maximum values of almost 0.44 are observed. Good correlations between the numerical estimations and experimental results indicate the validation of the FE model. However, a slight error of approximately $0.3-0.5 \%$ was found between the strain values. The root cause is a difference in a local coordinate system used in MatchID and the global system of Abaqus.
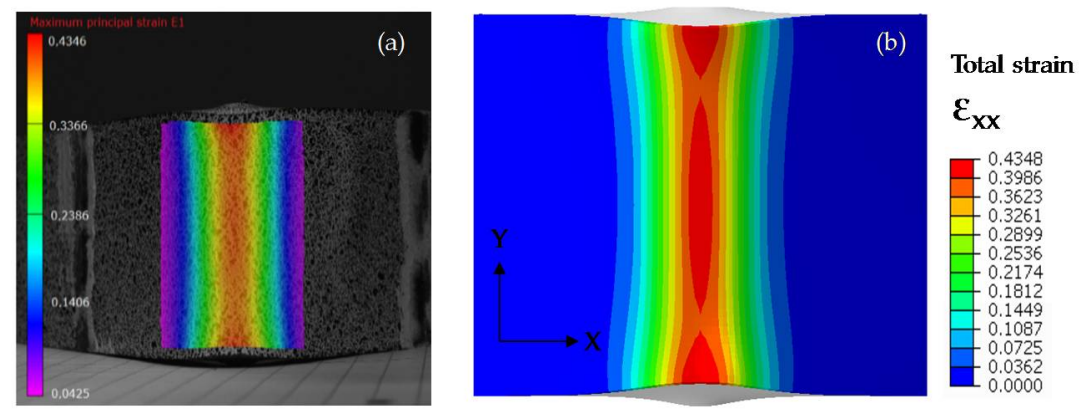

Figure 3. (a) DIC measurement of total strain $\varepsilon x x$ on bottom side; (b) Strain measured with FE-model.

\section{Results and Discussion}

After the bending process, the residual stress-strain distribution of the 3D solid model can be analysed. Figure 3 shows a comparison between the plastic strain derived from the experiment (a) and the simulation (b). From Figure $4 a$, it is clear that a substantial amount of more than 0.43 of strain can be measured along the bottom side of the deformed specimen, where values slightly increase towards the ends of the specimen since it is more concentrated in these areas. For fatigue testing, a tensile load perpendicular to the bend was applied shown in Figure 1c. This causes positive stress in the bending root that lead to crack initiation. The fracture surface of broken fatigued sample was used for fractography. The stress concentration inside of the bend increases due to tensile loading, resulting in crack initiation [7]. Residual shear strain on the crystallographic slip planes in combination with repeated crack opening conditions, induces persistent slip bands and fatigue striations in this area[3]. Because of a high stress amplitude, multiple crack initiation sites can be 
observed on the fracture surface shown in Figure 5. The formation of one dominant crack eventually caused the remaining cross-section to fail by tensile fracture.
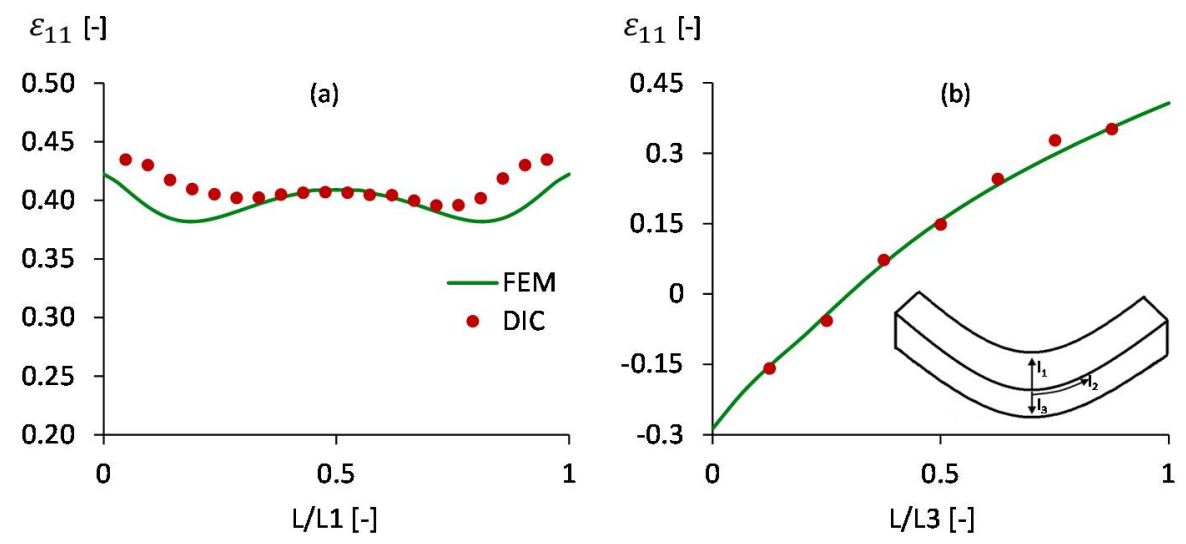

Figure 4. (a) Total strain $\varepsilon x x$ along bottom side; (b) Total strain $\varepsilon x x$ along front side.

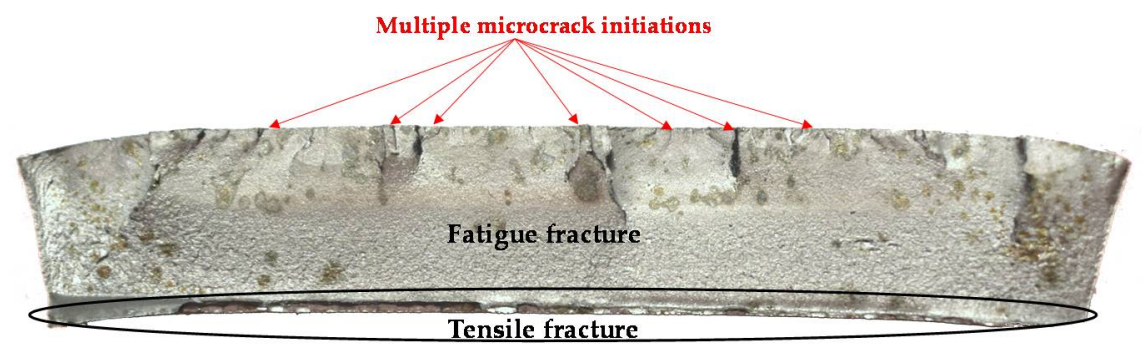

Figure 5. Fracture surface of V-fatigue sample.

The preliminary fatigue test, indicated the feasibility of the testing method for a different materials and thickness. Furthermore, complementary research is needed to attain a more complete characterisation and assess the scatter on fatigue data. Figure $6 \mathrm{~b}$ displays the evolution of the minimum and maximum displacement values and the amount of cycles spent in crack-initiation $(65 \%)$ and propagation (35\%). Propagation is defined experimentally when an increase of $0.1 \mathrm{~mm}$ from the stabilisation point (SP) is observed.

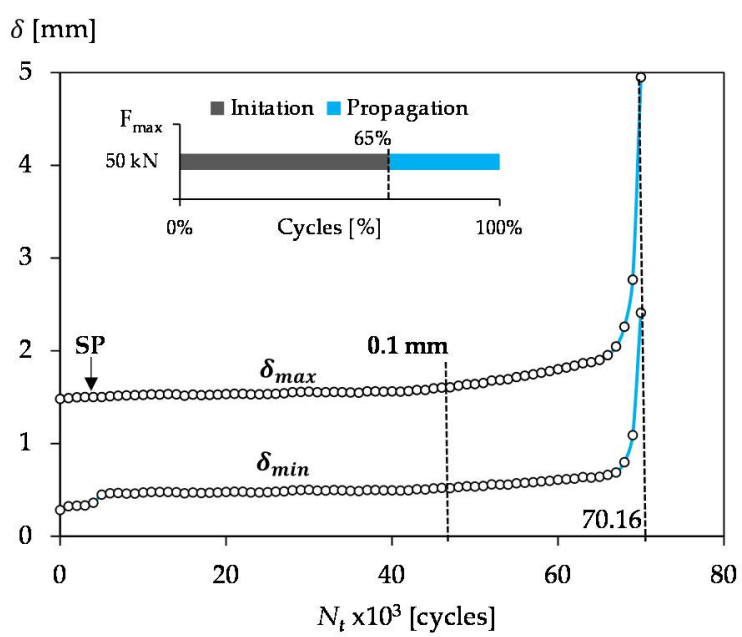

Figure 6. Displacement values of S690QL during fatigue testing and categorisation of fatigue life in \%. 


\section{Conclusions}

In the present paper, a bending process was simulated using numerical modelling. The developed model was then validated through a stereo DIC experiment. After the bending process, a fatigue test was performed, according to previous research by Talemi et al. [2] Future research will concern the application of DIC to trace the damage accumulation, during the fatigue testing, through incremental measurements at every $n$ load cycles. The study has primarily been concerned with the use of DIC as a validation of a FE analysis capable of predicting the residual stress induced by a forming process. Adopting a V-shaped bent fatigue test set-up, S690QL could be tested and analysed. However, to identify the cyclic behaviour of this HSS grade further, additional testing is required and a comparative analysis with similar materials can be conducted.

Author Contributions: S. Gothivarekar designed and performed the experiments with supervision of S. Coppieters, R. Talemi and D. Debruyne wrote the paper with revision of S. Coppieters, R. Talemi and D. Debruyne.

Acknowledgments: The authors would like to acknowledge MatchID for the use of software.

Conflicts of Interest: The authors declare no conflict of interest. The founding sponsors had no role in the design of the study; in the collection, analyses, or interpretation of data; in the writing of the manuscript, and in the decision to publish the results.

\section{References}

1. Azom: S690QL High Yield Structural Steel Properties and applications, 1 June 2010. Available online: www.azom.com. (accessed on 5 april 2018).

2. Talemi, R.H.; De Waele, W. Experimental and numerical study on effect of forming process on low-cycle fatigue behaviour of high-strength steel. FFEMS 2017, 40, 2050-2067.

3. Vucko, F.; Bosch, C. Experimental investigation of internal and effective stresses during fatigue loading of high-strength steel. J MSEA 2014, 597, 381-386, doi:10.1016/j.msea.

4. Shimatani, Y.; Shiozawa, K. The effect of the residual stresses generated by surface finishing methods on the VHCF behavior of matrix HSS. Int. J. Fatigue 2011, 33, 122-131, doi:10.1016/j.ijfatigue.

5. Denys, K.; Zhang, H.; Debruyne, D. Report on Basic Material Characterization Obtained with Homogeneous Specimens Only, no Through-Thickness Anisotropy or Inhomogeneity Included; KU Leuven: Gent, Belgium, 2017.

6. Sutton, M.; Orteu, J.J. Image Correlation for Shape, Motion and Deformation Measurements; Springer: New York, NY, USA, 2009; pp. 81-150.

7. Schijve, J. Fatigue of Structures \& Materials; Springer: Dordrecht, The Netherlands, 2009, pp. 12-134.

8. Denys, K. Investigation into the Plastic Material Behaviour of Thick HSS Using Multi DIC and FEMU. Ph.D. Thesis, KU Leuven, Gent, Belgium, 2017.

9. Coppieters, S.; Kuwabara, T. Identification of post-necking hardening phenomena in ductile sheet metal. Exp. Mech. 2014, 54, 1355-1371, doi:10.1007/s11340-014-9900-4. 